\title{
Ingestão de resíduos sólidos por tartarugas-verdes juvenis, Chelonia mydas (L. 1758), na costa leste do estado do Rio de Janeiro, Brasil
}

\author{
Danielle Rodrigues Awabdi ${ }^{1 *}$ \\ Salvatore Siciliano ${ }^{2}$ \\ Ana Paula Madeira Di Beneditto ${ }^{1}$

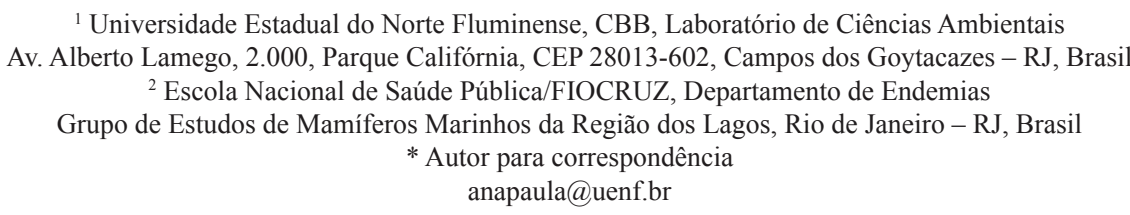

Submetido em 13/07/2012

Aceito para publicação em 06/11/2012

\section{Resumo}

Este trabalho analisou os resíduos sólidos de origem antropogênica obtidos em conteúdos estomacais de tartarugas-verdes juvenis, Chelonia mydas, na costa leste do estado do Rio de Janeiro, sudeste do Brasil (22 $2^{\circ} 50^{\prime} \mathrm{S}$ $\left.-23^{\circ} 00^{\prime} \mathrm{S}\right)$. Entre junho de 2009 e maio de 2010 foram analisados os conteúdos estomacais de 49 espécimes encalhados em praias. Resíduos sólidos foram registrados em 29 conteúdos estomacais (59,2\%) e verificou-se mais de uma categoria de resíduos em 22 amostras. Sacos e sacolas plásticas, categorizados como plásticos flexíveis, alcançaram a maior frequência de ocorrência (96,5\%). Provavelmente, esses resíduos se originaram a partir do descarte de sacos de lixo, sacos de ráfia, sacolas de estabelecimento comercial e embalagens diversas. Resíduos relacionados à confecção de artefatos de pesca também foram registrados com frequência, tais como fios de náilon, borracha, cordas, isopor e anzóis. A região é área de ocorrência regular de C. mydas e a ingestão relativamente elevada de resíduos sólidos depositados no ambiente representa risco à conservação dessa espécie.

Palavras-chave: Alimentação; Chelonia mydas; Ingestão; Resíduos sólidos; Sudeste do Brasil

\section{Abstract}

Ingestion of solid wastes by juvenile green turtles, Chelonia mydas (L. 1758), in the eastern Rio de Janeiro state coast, Brazil. This paper analyzed the solid wastes of anthropogenic origin obtained from the stomach contents of juvenile green turtles, Chelonia mydas, on the eastern Rio de Janeiro state coast, southeastern Brazil (22 $50^{\circ}$ S - 23 $\left.3^{\circ} 00^{\prime} S\right)$. Between June 2009 and May 2010, the stomach contents of 49 specimens stranded on beaches were analyzed. Solid wastes were recorded in 29 stomach contents $(59.2 \%)$ and more than one category of wastes was found out in 22 samples. Plastic bags, categorized as flexible plastic materials, achieved the greatest occurrence frequency (96.5\%). Perhaps, these wastes came from the disposal of garbage bags, raffia bags, commercial shop bags and various packaging. Wastes related to manufacture of fishing equipment were also often reported, such as nylon yarns, rubber, ropes, Styrofoam, and fishhooks. The region is an area of regular occurrence of C. mydas and the relatively large intake of solid wastes deposited in the environment poses a risk to the conservation of this species.

Key words: Chelonia mydas; Feeding; Ingestion; Solid wastes; Southeastern Brazil 
O despejo de resíduos de origem antropogênica em ambientes aquáticos ocorre há centenas de anos. Atualmente, os resíduos plásticos e derivados do petróleo são os principais poluentes dos oceanos registrados de forma visível (IVAR DO SUL; COSTA, 2007). Os resíduos sólidos descartados no ambiente marinho, tais como plásticos, restos de artefatos de pesca, vidro e borracha, são denominados de lixo marinho ("marine debris": em inglês).

Os vertebrados marinhos têm sofrido as consequências desse descarte, podendo ingerir os resíduos de modo acidental, se prender a eles ou devido a eles sofrer lesões que podem ser letais (BAKER et al., 2002; JAMES et al., 2005; GREG HOFMEYR et al., 2006; REIS et al., 2010). A ingestão de resíduos sólidos descartados no ambiente é fato bem descrito na literatura para as tartarugas marinhas (e.g., CARR, 1987; BJORDNAL, 1997; JAMES et al., 2005; REIS et al., 2010). Essa ingestão pode ocorrer ativamente, quando o resíduo é confundido com a fonte de alimento, ou passivamente quando é ingerido juntamente com o alimento (LAIST, 1987).

Bjordnal (1997) considera que a obstrução do trato gastrointestinal das tartarugas marinhas devido à ingestão de resíduos sólidos pode ocorrer mesmo quando pequenas quantidades são ingeridas, e que isso pode ser letal. A formação de fecalomas (massas de material fecal endurecido que obstruem o intestino) nesses animais é favorecida pela ingestão de resíduos sólidos. Outro problema decorrente dessa ingestão é a falsa sensação de saciedade, o que pode reduzir a frequência alimentar (LUTZ, 1990). A flutuabilidade das tartarugas marinhas pode ser afetada devido aos gases gerados após a ingestão e ao acúmulo dos resíduos sólidos no trato gastrointestinal (LAIST, 1987).

Diante do exposto, o presente estudo fornece as primeiras informações quantitativas sobre os resíduos sólidos ingeridos por espécimes juvenis de tartarugaverde, Chelonia mydas (L. 1758), na costa leste do estado do Rio de Janeiro (22 $50^{\circ} \mathrm{S}-23^{\circ} 00^{\prime} \mathrm{S}$ ) e busca identificar a provável origem desses resíduos.

Entre junho de 2009 e maio de 2010 foram coletados 49 espécimes juvenis de C. mydas a partir de encalhes em praias. Sete animais apresentavam sinais de interação com atividades de pesca a partir de marcas da malha da rede nas nadadeiras e cortes causados por fios de náilon. Os indivíduos foram medidos com fita métrica flexível $(0,1 \mathrm{~cm})$ em relação ao comprimento curvilíneo mínimo da carapaça, tomado a partir do ponto anterior médio do escudo nucal até o entalhe posterior médio dos escudos supracaudais. O estômago foi extraído da cavidade celomática após a remoção do plastrão. Utilizou-se peneira de $500 \mu \mathrm{m}$ de malha e água corrente para retirada dos itens presentes nos conteúdos estomacais. Os resíduos sólidos recuperados foram categorizados conforme a natureza do material e pesados em balança digital $(0,001 \mathrm{~g})$. Considerando os sacos e sacolas plásticas, categorizados como plástico flexível, a área superficial dos pedaços recuperados foi medida com fita métrica flexível $(0,1 \mathrm{~cm})$. A frequência de ocorrência (\%) das categorias de resíduos sólidos foi calculada dividindo-se o número de vezes em que uma categoria estava presente nos conteúdos estomacais pelo número total amostrado que continha resíduo (Tabela 1).

O comprimento curvilíneo mínimo da carapaça dos espécimes de $C$. mydas analisados variou entre 27,0 e 49,0 cm (média: 35,9 $\pm 6,5 \mathrm{~cm}$ ), caracterizando a sua condição juvenil (BJORDNAL, 1997). Resíduos sólidos foram registrados em 29 conteúdos estomacais (59,2\%). A presença de mais de uma categoria de resíduos foi observada em 22 amostras. A ocorrência de fecalomas no intestino foi constatada visualmente em cinco espécimes (17\%) os quais também continham resíduos sólidos no conteúdo estomacal.

A categoria dos plásticos apresentou a maior frequência de ocorrência nos conteúdos estomacais e a maior contribuição em peso total de resíduos sólidos, mas a contribuição dos resíduos provenientes de atividades de pesca também foi frequente (Tabela 1). A variação do peso dos resíduos sólidos recuperados em cada estômago foi de menos de $1 \mathrm{~g}$ a $58 \mathrm{~g}$, incluindo nesse intervalo os espécimes com registro de fecalomas. A área superficial dos pedaços de sacos e sacolas plásticas registradas variou de menos de $1 \mathrm{~cm}^{2}$ a $80 \mathrm{~cm}^{2}$.

Diversos autores relatam a elevada frequência (50$70 \%$ ) de ingestão de resíduos sólidos por C. mydas, com predominância de material plástico e fios de náilon, (e.g., Flórida, EUA: BJORNDAL et al., 1994; Rio Grande do 
TABELA 1: Resíduos sólidos registrados nos conteúdos estomacais de Chelonia mydas na costa leste do estado do Rio de Janeiro e sua provável origem (N: número de conteúdos estomacais com presença do resíduo sólido; FO: frequência de ocorrência; Peso: peso total da categoria de resíduo sólido em $\mathrm{N}$ conteúdos estomacais).

\begin{tabular}{|c|c|c|c|c|}
\hline Categoria & $\mathbf{N}$ & $\begin{array}{l}\text { FO } \\
(\%)\end{array}$ & $\begin{array}{c}\text { Peso } \\
(\mathrm{g})\end{array}$ & Provável origem do resíduo sólido \\
\hline \multicolumn{5}{|l|}{ 1) Plástico flexível (transparente, branco e colorido) } \\
\hline 1.a) Sacos e sacolas plásticas & 28 & 96,5 & 64,0 & $\begin{array}{l}\text { Sacos de lixo; sacos de ráfia; sacolas de } \\
\text { estabelecimentos comerciais; embalagens }\end{array}$ \\
\hline 1.b) Embalagens de doces e rótulos & 09 & 31,0 & 3,6 & Embalagens \\
\hline 1.c) Copos descartáveis e canudos & 02 & 6,9 & 0,8 & Copos descartáveis e canudos \\
\hline 2) Plástico rígido (transparente, branco e colorido) & 16 & 55,2 & 138,1 & Embalagens; tampas \\
\hline 3) Fios de náilon & 17 & 58,6 & 5,7 & Artefatos de pesca \\
\hline 4) Corda e barbante & 10 & 34,5 & 4,3 & Artefatos de pesca; embalagens \\
\hline 5) Tecido e fibra sintética & 07 & 24,1 & 1,1 & $\begin{array}{l}\text { Peças de vestuário, cama, mesa e/ou banho; } \\
\text { móveis e objetos de decoração }\end{array}$ \\
\hline 6) Borracha & 06 & 20,7 & 3,6 & $\begin{array}{l}\text { Pneus (incluindo atividades de pesca); } \\
\text { elástico; balões de festa; tampas }\end{array}$ \\
\hline 7) Isopor & 05 & 17,2 & 1,3 & $\begin{array}{l}\text { Artefatos de pesca, caixas de refrigeração, } \\
\text { embalagens }\end{array}$ \\
\hline 8) Diversos ${ }^{1}$ & 04 & 17,2 & 13,6 & Diversos \\
\hline 9) Papelão & 02 & 6,9 & 0,2 & Embalagens \\
\hline 10) Anzol & 01 & 3,4 & 0,2 & Artefatos de pesca \\
\hline
\end{tabular}

${ }^{1}$ Categoria inclui: lâmpada pisca-pisca, papel alumínio, itens não identificados.

Sul, Brasil: BUGONI et al., 2001; TOURINHO et al., 2010; Baía de Los Angeles, México: SEMINOFF et al., 2002; oceano Pacífico: BOYLE; LIMPUS, 2008; Paraná, Brasil: GUEBERT-BARTHOLO et al., 2011; Bahia, Brasil: MACEDO et al., 2011). Os resultados do presente estudo são comparáveis aos dos autores supracitados. No entanto, não estão sendo consideradas as possíveis diferenças entre os estudos em relação à metodologia de obtenção das amostras e à categorização dos resíduos sólidos.

A elevada frequência de resíduos plásticos nos conteúdos estomacais analisados pode estar relacionada à sua disponibilidade na área de estudo. Oigman-Pszczol e Creed (2007) realizaram levantamento sobre a ocorrência de resíduos antropogênicos nesta mesma área e verificaram que o material plástico e os restos de artefatos de pesca foram os resíduos sólidos submersos mais abundantes na região do infralitoral. A ingestão de resíduos sólidos por C. mydas, principalmente plásticos flexíveis e náilon, é favorecida por seu hábito alimentar e modo de apreensão do alimento. A espécie é preferencialmente herbívora, consumindo macroalgas e fanerógamas marinhas, e os sítios alimentares estão associados a áreas costeiras (BJORDNAL, 1997; BUGONI et al., 2001). O esqueleto apendicular das tartarugas marinhas é em forma de nadadeiras, não permitindo a seleção ou manipulação do alimento antes da ingestão (LUTZ; MUSICK, 1996). Essas características podem favorecer a ingestão acidental passiva de resíduos sólidos que provavelmente estão presos aos talos, apressórios e/ou superfície foliar das macroalgas e fanerógamas componentes da sua alimentação.

O grau de interação de $C$. mydas com resíduos sólidos na costa leste do estado Rio de Janeiro é relativamente elevado. Isso revela a necessidade de ações mais efetivas em relação à redução da produção e do uso de materiais sólidos descartáveis, principalmente de sacos e sacolas plásticas, que são lançados diretamente 
em áreas costeiras ou que alcançam essas áreas a partir da poluição de rios. A sociedade em geral deve ser orientada quanto à melhor forma de descarte dos resíduos sólidos, com incentivos à coleta seletiva e reciclagem. Adicionalmente, as comunidades pesqueiras também deveriam ser esclarecidas quanto à melhor forma de descarte de artefatos de pesca, minimizando esse tipo de poluição e consequentemente o risco de sua interação com as tartarugas-verdes. Essas medidas contribuiriam para a conservação da espécie e a manutenção da qualidade e saúde dos ecossistemas marinhos costeiros.

\section{Agradecimentos}

À equipe do Grupo deEstudos de Mamíferos Marinhos da Região dos Lagos (GEMM - Lagos) pela coleta dos espécimes de tartaruga-verde e cessão do conteúdo estomacal para a realização deste estudo; a bióloga Larissa Araújo Nunes pelo auxílio na triagem dos resíduos sólidos analisados; e ao CENPES/PETROBRAS que coordenou o 'Projeto Habitats: Heterogeneidade Ambiental da Bacia de Campos' envolvido neste estudo. D.R. Awabdi recebeu bolsa de mestrado da Coordenação de Aperfeiçoamento de Pessoal de Nível Superior - CAPES. A.P.M. Di Beneditto recebeu fomento do Conselho Nacional de Desenvolvimento Científico e Tecnológico - CNPq (300241/09-7) e da Fundação de Amparo a Pesquisa do Estado do Rio de Janeiro - FAPERJ (E-26/102.915/2011), e é membro do CNPq-INCT Transferência de Material Continente-Oceano (573.601/08-9).

\section{Referências}

BAKER, G. B.; GALES, R.; HAMILTON, S.; WILKINSON, V. Albatrosses and petrels in Australia: a review of their conservation and management. Emu, Collingwood, v. 102, n. 1, p. 71-96, 2002. BJORNDAL, K. A. Foraging ecology and nutrition of sea turtles. In: LUTZ, P. L; MUSICK, J. A. (Ed.). The biology of sea turtles. Boca Raton: CRC Press, 1997. p. 199-231.

BJORNDAL, K. A.; BOLTEN, A. B.; LAGUEUX, C. J. Ingestion of marine debris by juvenile sea turtles in Coastal Florida habitats. Marine Pollution Bulletin, Amsterdam, v. 28, n. 3, p. 154-158, 1994.

BOYLE, M. C.; LIMPUS, C. J. The stomach contents of posthatchling green and loggerhead sea turtles in the southwest Pacific: an insight into habitat association. Marine Biology, Kiel, v. 155, n. 2, p. 233-241, 2008.
BUGONI, L.; KRAUSE, L.; PETRY, M. V. Marine debris and human impacts on seaturtles in southern Brazil. Marine Pollution Bulletin, Amsterdam, v. 42, n. 12, p. 1330-1334, 2001.

CARR, A. Impact of nondegradable marine debris on the ecology and survival outlook of sea turtles. Marine Pollution Bulletin, Amsterdam, v. 18, n. 6B, p. 352-356, 1987.

GREG HOFMEYR, G. J.; BESTER, M. N.; KIRKMAN, S. P.; KOVACS, K. M.; LYDERSEN, C. Entanglement of Antarctic fur seals at Bouvetoya, Southern Ocean. Marine Pollution Bulletin, Amsterdam, v. 52, n. 9, p. 1077-1080, 2006.

GUEBERT-BARTHOLO, F. M.; BARLETTA, M.; COSTA, M. F.; MONTEIRO FILHO, Y. E. L. A. Using gut contents to assess foraging patterns of juvenile green turtles Chelonia mydas in the Paranaguá Estuary, Brazil. Endangered Species Research, London, v. 13, n. 2, p. 131-143, 2011.

IVAR DO SUL, J. A.; COSTA, M. F. Marine debris review for Latin America and the wider Caribbean region: from the 1970s until now, and where do we go from here? Marine Pollution Bulletin, Amsterdam, v. 54, n. 8, p. 1087-1104, 2007.

JAMES, M. C.; MYERS, R. A.; OTTENSMEYER, C. A. Identification of high-use habitat and threats to leatherback sea turtles in northern waters: new directions for conservation. Ecology Letters, Ontario, v. 8, p. 195-201, 2005.

LAIST, D. W. Overview of the biological effects of lost and discarded plastic debris in the marine environment. Marine Pollution Bulletin, Amsterdam , v. 18, n. 6B, p. 319-326, 1987.

LUTZ, P. L. Studies on the ingestion of plastic and latex by sea turtles. In: PROCEEDINGS OF THE WORKSHOP ON THE FATE AND IMPACT OF MARINE DEBRIS, 1990, Miami. NOAA Technical Memorandum NMFS. Honolulu: PROCEEDINGS OF THE WORKSHOP ON THE FATE AND IMPACT OF MARINE DEBRIS, 1985. Versão eletrônica.

LUTZ, P. L.; MUSICK, J. A. The biology of sea turtle. Boca Raton: CRC Press, 1996. 448 p.

MACEDO, G. R.; FRANKE, C. R.; GARCEZ NETO, A. F.; GOLDBERG, D. W.; LEAL, D. C.; PIRES, T. T.; ROSTÁN, G. Ingestão de resíduos antropogênicos por tartarugas marinhas no litoral norte do estado da Bahia, Brasil. Ciência Rural, Santa Maria, v. 41, n. 11, p. 1938-1941, 2011.

OIGMAN-PSZCZOL, S. S.; CREED, J. C. Quantification and classification of marine litter on beaches along Armação dos Búzios, Rio de Janeiro, Brazil. Journal of Coastal Research, West Palm Beach, v. 23, n. 2, p. 421-428, 2007.

REIS, E. C.; LIMA, L. M.; PEREIRA, C. S.; RENNÓ, B.; RODRIGUES, D. P.; SECCO, H. K. C.; SICILIANO, S. Condição de saúde das tartarugas marinhas do litoral centro-norte do estado do Rio de Janeiro, Brasil: avaliação sobre a presença de agentes bacterianos, fibropapilomatose e interação com resíduos antropogênicos. Oecologia Australis, Rio de Janeiro, v. 14, n. 3, p. 756-765, 2010.

SEMINOFF, J. A.; HIDALGO, S.; NICHOLS, W. J.; RESENDIZ, A. Diet of the East Pacific green turtle (Chelonia mydas) in the central Gulf of California, México. Journal of Herpetology, Washington, v. 36, n. 3, p. 447-453, 2002.

TOURINHO, P. S.; FILLMANN, G.; IVAR DO SUL, J. A. Is marine debris ingestion still a problem for the coastal marine biota of southern Brazil? Marine Pollution Bulletin, Amsterdam, v. 60, n. 3, p. 396-4010, 2010. 